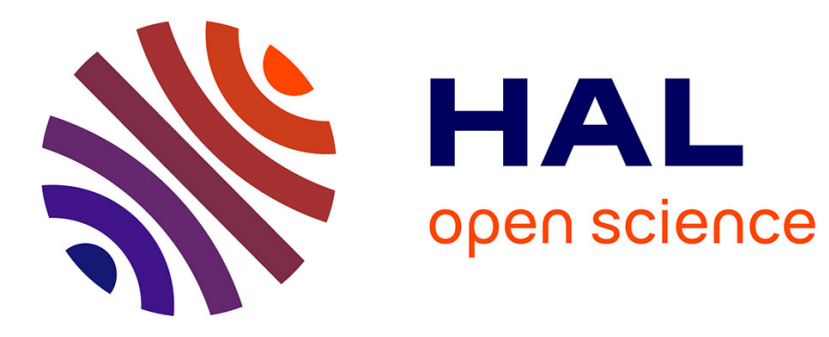

\title{
Age-related vulnerability of pattern separation in C57BL/6J mice
}

\author{
Aurélia Cès, Thibaut Burg, Karine Geiger, Céline Heraud, Jean-Bastien Bott, \\ Ayikoe-Guy Mensah-Nyagan, Chantal Mathis
}

\section{- To cite this version:}

Aurélia Cès, Thibaut Burg, Karine Geiger, Céline Heraud, Jean-Bastien Bott, et al.. Age-related vulnerability of pattern separation in C57BL/6J mice. Neurobiology of Aging, 2018, 62, pp.120-129. 10.1016/j.neurobiolaging.2017.10.013 . hal-02373365

\section{HAL Id: hal-02373365 https://hal.science/hal-02373365}

Submitted on 20 Nov 2019

HAL is a multi-disciplinary open access archive for the deposit and dissemination of scientific research documents, whether they are published or not. The documents may come from teaching and research institutions in France or abroad, or from public or private research centers.
L'archive ouverte pluridisciplinaire $\mathbf{H A L}$, est destinée au dépôt et à la diffusion de documents scientifiques de niveau recherche, publiés ou non, émanant des établissements d'enseignement et de recherche français ou étrangers, des laboratoires publics ou privés. 


\section{Age-related vulnerability of pattern separation in $\mathrm{C} 57 \mathrm{BL} / 6 \mathrm{~J}$ mice}

Aurélia Cès ${ }^{\mathrm{a}}$, Thibaut Burg ${ }^{\mathrm{a}}$, Karine Herbeaux ${ }^{\mathrm{a}}$, Céline Héraud ${ }^{\mathrm{a}}$, Jean-Bastien Bott ${ }^{\mathrm{a}}$, Ayikoe Guy Mensah-Nyagan ${ }^{b}$, Chantal Mathis ${ }^{a}$

a Université de Strasbourg, CNRS UMR 7364, Laboratoire de Neurosciences Cognitives et Adaptatives (LNCA), 12 Rue Goethe, Strasbourg, France

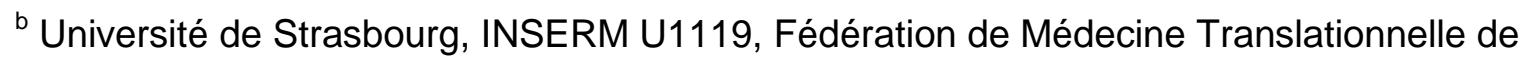
Strasbourg (FMTS), Biopathologie de la Myéline, Neuroprotection et Stratégies

Thérapeutiques, Bâtiment 3 de la Faculté de Médecine, 11 Rue Humann, Strasbourg, France

Corresponding author:

Chantal Mathis, PhD

Université de Strasbourg, Laboratoire de Neurosciences Cognitives et Adaptatives, CNRSUMR7364, 12 Rue Goethe, F-67000 Strasbourg, France

Tel.: + 33368851876

Fax: + 33368851958

e-mail: chantal.mathis@unistra.fr

key-words: Cognitive aging; Pattern separation; Object location; Object feature; D-serine 
Abstract

Aging is associated with impaired performance in behavioral pattern separation (PS) tasks based on similarities in object features and in object location. These deficits have been attributed to functional alterations in the dentate gyrus (DG)-CA3 region. Animal studies suggested a role of adult-born DG neurons in PS performance. The present study investigated the effect of aging in C57BL/6J mice performing PS tasks based on either object features or object location. At the age of 18 months or more, performance was severely impaired in both tasks. Spatial PS performance declined gradually over adult lifespan from 3 to 21 months. Subchronic treatment with the cognitive enhancer D-serine fully rescued spatial PS performance in 18-month-old mice and induced a modest increase in the number of 4-week-old adult-born cells in the DG. Performance of mice in these PS tasks shows an age-dependence which appears to translate well to that found in humans. This model should help in deciphering physiological changes underlying PS deficits and in identifying future therapeutic targets. 


\section{Introduction}

In everyday life, we often have similar experiences in similar contexts. Thus, the ability to discriminate individual experiences based on specific memories appears particularly useful in daily activities (Conway, 2009). In humans and animals, aging is known to affect performance in tasks evaluating the ability to detect subtle changes in object position or features between successive sample and test sessions (e.g., Reagh et al., 2014; Stark et al., 2010; Toner et al., 2009). These tasks are often called behavioral pattern separation (PS) tasks, in reference to the pattern separation computational model. This model defines a system capable of orthogonalizing overlapping inputs into non-overlapping outputs which could potentially resolve interference between similar memories (Marr, 1971; Rolls and Kesner, 2006). Separation-like fMRI activity related to PS performance was reported in medial temporal structures, especially within the dentate gyrus (DG)-CA3 region (Bakker et al., 2008; Reagh and Yassa, 2014). Impaired PS performance in the elderly correlated with altered DG-CA3 activation and reduced the integrity of medial temporal lobe connectivity (Lacy et al., 2010; Yassa et al., 2011).

An involvement of the DG-CA3 region in behavioral PS was also repeatedly shown in animal studies using various experimental approaches, from lesions to genetic manipulation (e.g., Deng et al., 2013; Gilbert et al., 2001; Goodrich-Hunsaker et al., 2008; Leutgeb et al., 2007). Manipulation of the level of dentate adult neurogenesis and changes in dentate $N$-methyl-Daspartate receptor (NMDAR) functionality was shown to affect memory performance in PS tasks based on object features, spatial location or context (e.g., Bolz et al., 2015; Clelland et al., 2009; Creer et al. 2010; Kannangara et al., 2014; McHugh et al., 2007; Sahay et al. 2011; Tronel et al., 2012). However, it must be noted that the role of adult-born DG neurons in a PS-like computation within the DG has often been questioned (Deng et al., 2013; Groves et al., 2013; Becker, 2016). Surprisingly, despite a growing literature investigating the neuronal basis of behavioral PS in adult animals and extensive evidence for its high sensitivity to aging in humans, only a few studies investigated PS in aged animals (e.g., Creer et al. 2010; Gracian et al., 2013; Wu et al., 2015; Marrone et al., 2011). The rare attempts to restore PS 
performance in aged mice were based on the neurogenesis hypothesis, and resulted in mixed outcomes. Exercise provided only a moderate improvement in PS performance in 22month-old mice and their level of DG neurogenesis remained very low, whereas it boosted both performance and neurogenesis in 3-month-old mice (Creer et al, 2010). Wu et al. (2015) showed that exercise completely overcame severe PS deficits in 18-month-old mice performing a contextual discrimination task, but with only a slight increase in the number of new DG neurons.

The first aim of the present study was to characterize the effect of age on PS performance in C57BL/6J mice. We developed two novelty detection tasks based on object features and object location respectively, using a sample trial and a test trial in order to be as close as possible to the computer-based tasks used in humans (Stark et al., 2013; Reagh et al., 2014). The second aim was to restore spatial PS performance in aged mice by means of Dserine, an endogenous co-agonist of NMDAR known to reverse age-dependent impairment of synaptic plasticity and cognitive performance in rodents and humans (Mothet et al., 2015; Avellar et al., 2016; Levin et al., 2015). Survival of new-born DG cells was subsequently quantified to examine whether D-serine modulates neurogenesis in aged mice (Sultan et al., 2013).

\section{Material and methods}

\subsection{Animals}

C57BL/6J male mice were obtained from Charles River Laboratories (France). Procedures were in compliance with rules of the European Community Council Directive 2010-63 and French Department of Agriculture Directive 2013-118, and approved by the local review board (CREMEAS: AL/14/21/02/13, AL/11/18/02/13 and APAFIS\#2154). Animal facilities were approved for animal experimentation (H 67-482-13). Upon arrival, mice were grouphoused with food and water ad libitum, nesting material and additional food pellets on bedding to promote natural behavioral patterns. The animal room was under controlled temperature $\left(23 \pm 1^{\circ} \mathrm{C}\right)$ and a 12/12-hour light/dark cycle (lights on at 8.00 am). Behavioral 
testing took place during the light phase. Mice were single-housed for 1 week before testing, due to a possible age-dependent confounding factor detected in previous pattern discrimination studies: when unrelated C57BL/6J males were obtained at different ages, as in the present study, a large subset of 5-to-12-month-old mice showed systematic aggressive behavior, especially towards mice returning to the home cage after habituation and discrimination sessions. C57BL/6J male mice fighting in the home cage is well documented, and can cause variations in behavior and brain function (e.g., Greenberg et al., 2014). General health status was checked with particular attention to exclude vibrissae alterations (barbering) and eye abnormalities (e.g., cataract or closed eye) liable to bias PS performance.

\subsection{Spatial pattern separation task}

Rodents have a spontaneous tendency to preferentially re-explore an object displaced within a familiar context (Ennaceur et al., 1997). In principle, PS ability should be evaluated with object displacement close to the limits of behavioral detection. The task began with one or two daily 10-min habituation sessions in the presence of two identical objects, never experienced before, each positioned $30 \mathrm{~cm}$ from the walls on the midline of the open field (92.5 x $92.5 \times 35 \mathrm{~cm}$; Ugo Basile, Italy; 30 lux in the center). On the following day(s), the mice received a daily session consisting of a sample trial (duration specified for each experiment), then 4 minutes in the home cage, and a 6-min test trial. For each sample-test session, a new pair of identical objects was positioned on the diagonal of the open field (see Figure 1a). For the sample trial, the fixed object was always $37 \mathrm{~cm}$ from the corner and the second object $20 \mathrm{~cm}$ away from the fixed object. During the test trial, the second object could be moved $10,15,20,25,30$ or $35 \mathrm{~cm}$ further along the diagonal. Object exploration was defined as active exploration with the nose pointing toward an object within $2 \mathrm{~cm}$. Sitting on or next to or licking or gnawing the object was not considered as exploration. Equal interest for both objects during sample trials was systematically checked for each group (i.e., absence of location preference). Spatial discrimination performance was evaluated as time spent exploring the displaced object as a percentage of total exploration time for the two 
objects during the first $3 \mathrm{~min}$ of the test trial. This performance was expected to be higher than the $50 \%$ chance level if the mouse detected the object displacement. For the last $3 \mathrm{~min}$ of the test trial, performance was lower and closer to chance level, suggesting that mice expressed novelty detection behavior mainly during the first $3 \mathrm{~min}$. Objects (5 to $6.5 \mathrm{~cm}$ diameter, 4.5 to $7 \mathrm{~cm}$ high) composing each pair were selected so as to induce similar exploration times in preliminary studies: a napkin ring, a candlestick and an egg-cup, each topped with marbles of different size and color. Habituation objects were less than $10 \mathrm{~cm}$ in height and diameter.

\subsection{Object pattern separation task}

Rodents also have a tendency to explore preferentially a new rather than a familiar object in a familiar context (Ennaceur and Delacour, 1988). Object PS ability was evaluated using a new object that was very similar to the familiar one. Two groups of mice (4 and 18 months old) were familiarized for two days (10 min/day) with the open field (52 x 52 × $40 \mathrm{~cm}$; 40 lux in the center) in presence of two objects, renewed each day. The next two days, the daily session consisted of a sample trial with a pair of identical objects, followed by 4 minutes in the home cage, and a 10-min test trial with one of the pair of objects replaced by a new one that was either very similar or not. The two objects were always positioned in the same adjacent corners (10 $\mathrm{cm}$ from the walls). Absence of side preference was checked for each group during sampling. The position of the new object and its level of similarity were counterbalanced over groups and days. Two sets of objects with different levels of similarity were used, one for each test day. Each triplicate of familiar objects was constructed from Lego ${ }^{\circledR}$ bricks, with a fixed number of bricks per color. For each set of familiar objects, an identical set of bricks was used to construct two novel objects with exactly the same shape as the familiar objects, but one with high and the other with low similarity. All objects had an identical gray Lego ${ }^{\circledR}$ sheet as a base, which could be secured to the floor with Patafix ${ }^{\circledR}$ (UHU). Performance was evaluated as the percentage of total exploration time for the two objects spent exploring the new object during the whole test trial. All other procedures were similar to those described above. 


\section{Spatial navigation tasks}

As aged mice showed profound deficit in the spatial PS task, we checked whether the same mice would also show deficits in other spatial tasks routinely used in our laboratory, such as the Morris water-maze task or the Barnes maze task (Moreau et al., 2008). Two subsets of young and old mice evaluated in the spatial PS task (one per experiment) were subsequently tested on one or other of these tasks (Supplementary Material and Methods).

\subsection{Drug administration, immunohistochemistry and image analysis}

In the last experiment, all mice received three intraperitoneal (IP) injections of 5-bromo-2deoxyuridine (BrdU; Sigma-Aldrich; diluted in $0.1 \mathrm{M}$ phosphate buffer, $\mathrm{pH} 8.4,40^{\circ} \mathrm{C}, 100$ $\mathrm{mg} / \mathrm{kg} /$ injection, $0.1 \mathrm{~mL} / \mathrm{kg} /$ injection) at $9.00 \mathrm{am}, 11.00 \mathrm{am}$ and $2.00 \mathrm{pm}$ in a single day, exactly 28 days before testing spatial PS performance. This 4-week interval was based on studies suggesting a role of 3-to-4-week-old DG neurons in spatial and contextual PS performance (Imielski et al., 2012; Kheirbek et al, 2012; Nakashiba et al., 2012). For D-serine (Sigma-Aldrich; diluted in $0.9 \% \mathrm{NaCl}$ solution), we selected a subchronic $50 \mathrm{mg} / \mathrm{kg}$ IP treatment known to improve both memory performance and survival of new DG neurons in mice (Bado et al., 2011; Filali and Lalonde, 2013; Sultan et al., 2013). Mice were injected with D-serine or vehicle $(0.1 \mathrm{~mL} / \mathrm{kg})$ once per day $(9: 00 \mathrm{am})$ for seven consecutive days beginning 18 days after BrdU injection and completed the day before the drug-free test period. This treatment period (days 18-24) corresponded to a critical period of new-born DG neuron maturation and survival (Snyder et al., 2009; Veyrac et al., 2013). Treatment was applied a few days earlier than in the 28-day protocol used by Sultan et al. (2013), as a pilot study showed that repeated injection during the test period had a deleterious effect on PS performance.

One day after testing, mice were deeply anesthetized $(120 \mathrm{mg} / \mathrm{kg}$ pentobarbital, $10 \mathrm{~mL} / \mathrm{kg}, \mathrm{IP})$ before intracardiac perfusion with phosphate-buffered saline (PBS, $0.1 \%$ heparin), followed by $4 \%$ paraformaldehyde solution (Carl Roth $\mathrm{GmbH}+$ Co. KG, Germany; 0.1 M phosphate buffer, $\left.\mathrm{pH} 7.4,4^{\circ} \mathrm{C}\right)$. Brains were postfixed $24 \mathrm{~h}$ in $4 \%$ PFA $\left(4^{\circ} \mathrm{C}\right)$ and stored in PBS $\left(4^{\circ} \mathrm{C}\right)$. $50-\mu \mathrm{m}$ coronal sections (Leica Biosystems vibratome) were stored free-floating at $-20^{\circ} \mathrm{C}$ in a 
cryostorage solution (30\% glycerol, 30\% ethylene glycol, $0.026 \mathrm{M}$ phosphate buffer). For immunostaining, sections were PBS-rinsed and incubated 15 min in $2 \mathrm{~N} \mathrm{HCl}$ at $37^{\circ} \mathrm{C}$. After washing, they were incubated in $0.3 \% \mathrm{H}_{2} \mathrm{O}_{2}$ in methanol (30 min, room temperature) to quench endogenous peroxidase activity, then extensive rinsing was followed by blocking in $5 \%$ normal goat serum in PBS- $0.5 \%$ Triton $\mathrm{X} 100$ before incubation in monoclonal rat antiBrdU antibody (1/500, Abcam) for $24 \mathrm{~h}$ at room temperature. After several washes, sections were incubated with biotinylated rabbit anti-rat (1/500, Vector Laboratories; $2 \mathrm{~h}$, room temperature) followed by $30 \mathrm{~min}$ with avidin-biotin-peroxidase complex (Vector Laboratories, Burlingame, California, USA) and staining development at room temperature with 3,3'-diaminobenzindine (Vectastain ABC kit and DAB kit, Vector Laboratories, Burlingame, California, USA).

BrdU-positive $\left(\mathrm{BrdU}^{+}\right)$cells were quantified in the granule cell layer $(\mathrm{GCL})$, including the subgranular zone (defined as a 2-cell-body-wide zone bordering the GCL and the hilus) of the dorsal DG. Counting was performed through the entire section thickness (40x objective) in every fifth section (50 $\mu \mathrm{m}$ thick, $250 \mu \mathrm{m}$ apart) spanning $-0.94 \mathrm{~mm}$ (first section) to -2.18 $\mathrm{mm}$ from the Bregma ( 5 sections per mouse). The fifth section was lost for two young mice. The experimenter was blind to treatment, but the mouse's age could easily be guessed. The GCL area was measured for each section with ImageJ software on DG images captured with the 4x objective of an Olympus AHBT3 microscope (Schneider et al., 2012). GCL volume for each section was obtained by multiplying total GCL area (left GCL + right GCL) by section thickness $(50 \mu \mathrm{m})$. For each mouse, $\mathrm{BrdU}^{+}$cell density was calculated by dividing total $\mathrm{BrdU}^{+}$ cell count by the total volume of GCL screened.

\subsection{Statistical analyses}

Discrimination and navigation performances were analyzed on 1-way ANOVA (Group) with repeated-measures on one or more factors (Distance, Distance Category, Day, Object, Order), followed by post hoc Newman-Keuls (NK) test. The performance of each group on each task was compared to chance on Student $t$ test. The Spearman rank correlation test was used to check whether sampling exploration or $\mathrm{BrdU}^{+}$cell density correlated with PS 
performance. A Student $t$ test was used in the last experiment to compare $\mathrm{BrdU}^{+}$cell densities between age groups. Differences were considered significant at $p<0.05$. Data are given as mean \pm SEM.

\section{Results}

\subsection{Minimal object displacement detected by 6-month-old mice}

After a 2-day familiarization period, two groups of 6-month-old mice were tested on one of each distance category (short, medium or long) over three days (group A: 10, 20 and $30 \mathrm{~cm}$, $\mathrm{n}=13$; group $\mathrm{B}: 15,25$ and $35 \mathrm{~cm}, \mathrm{n}=16$, Figure 1a). The order of the three distance categories was counterbalanced over groups and days.

Globally, group B explored objects $(20.9 \pm 0.9 \mathrm{~s})$ slightly less than group A $(25.0 \pm 1.4 \mathrm{~s})$ during the 12-min sample trial $(F(1,27)=5.63, p=0.025$, with no effect of Distance Category or Distance Category x Group interaction). The apparent significant difference seems to have been due to an outlier: when one mouse (group A) with very long sampling time $(60.1 \mathrm{~s})$ is removed from analysis, the group effect is no longer significant.

During the test trial, preference for the displaced object increased as a function of Distance Category $(F(2,54)=43.88, p<0.0001$, with no Group effect or interaction with Distance Category; Figure 1b). Both groups detected changes for medium and long distances only (from $20 \mathrm{~cm}$ onwards). The intermediate level of $20-\mathrm{cm}$ performance indicated that discrimination was particularly challenging at this distance, which was therefore subsequently used to test spatial PS performance. The amount of object exploration during the sampling trial did not influence subsequent performance on the medium Distance Category (Spearman rank test. group $A: \rho=-0.42, z=1.45, p=0.15$; group $B: \rho=-0.40, z=1.56, p=0.12$ ). Performances were not sensitive to the Order of Distance Categories (Group x Distance Category $x$ Order: $F(10,34)=0.29, p>0.10$; no effect of Order or interaction with Order; data not shown).

\subsection{Spatial pattern separation performance in 20-month-old mice}


After a 2-day habituation period, a new batch of 6 - and 20-month-old mice ( $\mathrm{n}=7 /$ group) were tested at the shortest, longest and most challenging distances $(10,35$ and $20 \mathrm{~cm}$, respectively; Figure 2a) in pseudo-randomized order over a 3-day period. As old mice often show short exploration times, the sample trial was increased from 12 to 15 min for all the following experiments.

Despite the extended duration, old mice explored less than young ones during sampling for all distances (young: $45.1 \pm 2.5$ s; old: $31.1 \pm 2.3$ s; Age effect: $F(1,24)=15.53, p=0.002$; no Distance effect or interaction with Age).

During the test trial, performances on the three distances varied as a function of age (Age $x$ Distance: $F(2,24)=3.58, p=0.04$; Distance effect: $F(2,24)=10.1, p=0.0007$; no Age effect: $F(1,24)=1.06, p=0.32$; Figure $2 b)$. Both groups did not detect the $10-\mathrm{cm}$ displacement, but easily detected the $35-\mathrm{cm}$ displacement. At the challenging $20-\mathrm{cm}$ distance, younger mice performed very well (NK post-hoc: $p<0.0510-\mathrm{cm}$ vs. $20-\mathrm{cm}$ distance), whereas old mice performed close to chance level and very poorly compared to young mice (NK post-hoc: $p<$ 0.05 young vs. old at $20-\mathrm{cm}$ distance, not significant at 10 - and $35-\mathrm{cm}$ distances). There was no significant correlation between sampling exploration and $20-\mathrm{cm}$ performance (Spearman rank test. All mice: $\rho=0.25, z=0.91, p=0.36$; young mice: $\rho=-0.25, z=0.61, p=0.54$; old mice: $\rho=-0.71, z=1.75, p=0.08)$.

After a 7-day rest period, the spatial navigation performance of the same mice was determined in a Barnes maze task. Old mice performed as well as young mice during the training phase (Figure S1a) and the 28-day delayed probe test (Figure S1b).

\subsection{Age-dependent evolution of spatial pattern separation performance}

The above results suggested that spatial PS performance was affected by normal aging. We therefore characterized age-related changes in 20 -cm performance using groups of different ages: 3, 7, 11, 16 and 21 months (Figure 2b).

To limit a possible impact of age-dependent variation in sampling exploration on subsequent discrimination performance (as noted previously), mice exploring less than $15 \mathrm{~s}$ or more than $40 \mathrm{~s}$ during the 15 -min sampling trial were discarded from the data set $(1<15 \mathrm{~s}$ at 3 months, 
final $n=13$, sampling exploration: $25.6 \pm 2.5 \mathrm{~s} ; 2>40 \mathrm{~s}$ at 7 months, final $n=13$, sampling exploration: $29.8 \pm 1.8 \mathrm{~s} ; 2>40 \mathrm{~s}$ at 11 months, final $\mathrm{n}=12$, sampling exploration: $29.1 \pm 2.1$ $\mathrm{s} ; 2<15 \mathrm{~s}$ at 16 months, final $\mathrm{n}=11$, sampling exploration: $22.2 \pm 1.0 \mathrm{~s} ; 3<15 \mathrm{~s}$ and $1>40 \mathrm{~s}$ at 21 months, final $n=11$, sampling exploration: $27.2 \pm 1.9 \mathrm{~s}$ ). There was no significant difference in sampling exploration between groups $(F(4,55)=2.29, p=0.071)$.

During the test trial, performance was significantly affected by age $(F(4,55)=5.92, p=$ 0.0005). The deficit appeared around middle age, as the 3-month-old group outperformed all groups aged 11 months and over. At the ages of 16 and 21 months, mice performed close to chance level. There was no significant correlation between sampling exploration and PS performance (Spearman rank test. All groups: $\rho=-0.14, z=1.11, p=0.26$, each individual group: $-0.59 \leq \rho \leq 0.05, z \leq 1.87, p>0.05)$.

After a 7-day rest period, subgroups of the 7- and 21-month-old mice tested above were submitted to a classical version of the spatial water-maze task; older mice performed as well as younger ones during the training (Figure S1c) and 7-day delayed probe test (Figure S1d).

\subsection{Object pattern separation performance in 18-month-old mice}

As aging appeared to affect spatial PS performance, we tested young and old mice (4 and 18 months old, $n=13$ /group) in a different task designed to evaluate object PS performances using two sets of objects with different levels of similarity (Figure 3a).

The 15-40 s criterion used previously still left some doubt as to a possible impact of agedependent variation in sampling exploration on subsequent PS performance. Each old mouse was therefore paired with a young one, so that the sample trial of the young mouse was terminated after 10 min or when it reached the total object exploration time performed by the paired old mouse during the 10-min sampling trial (young mice: $9.0 \pm 0.5 \mathrm{~s}$; old mice: 9.4 \pm 0.6 s; Age x Day: $F(1,24)=0.03, p>0.10$; no effect of Age or Day).

During the test trial, object discrimination performances were globally affected by age $(F(1,24)=13.0, p<0.002)$ and object similarity $(F(1,24)=22.83, p<0.0001)$. More importantly, the age effect on performance depended on the level of similarity of the novel object (Age x Object: $F(1,24)=6.52, p<0.02)$ (Figure 3b). Young mice showed a significant 
preference for the novel object, whatever its level of similarity with the familiar one; old mice discriminated novel objects with low similarity as well as young mice, but their performance dropped to chance level for objects with high similarity.

In general, levels of performance appeared slightly lower in the object task than in the spatial task. In the object PS task, it rapidly became clear that making stepwise changes in Lego arrangements was less straightforward than changing the distance between two objects: mice showed more graded reactions in relation to graded spatial changes. Therefore, Dserine was tested in the more flexible spatial PS task, to increase the chance of detecting beneficial effects in aged mice.

\subsection{Effect of D-serine on spatial pattern separation performance in 18-month-old mice} The effects of D-serine were tested in 18-month-old mice as Wu et al. (2015) showed that complete rescue of PS performance was possible at this age. Daily injections of D-serine (50 $\mathrm{mg} / \mathrm{kg}, \mathrm{IP}$ ) or vehicle were given during the week preceding behavioral testing (Figure 4a). Groups were constituted of 3-month-old mice treated with vehicle $(n=11)$ and 18-month-old mice treated with vehicle or $D$-serine ( $n=11$ /group). The group of 3-month-old mice was used as an internal control for task reliability in case D-serine had no effect, and also to compare the performance of the two older groups against an optimal level of performance (see Figure 2b). One old vehicle-treated mouse was eliminated as it did not explore the objects. During the second day of habituation, mice were tested on a $0-\mathrm{cm}$ distance test to improve familiarization with the test schedule and to check that they would explore both objects equally when there was no change (young vehicle: $48.2 \pm 4.0 \%$; old vehicle: $44.6 \pm 2.6 \%$; old D-serine: $48.9 \pm 2.8 \% ; F(2,29)=0.51, p>0.10)$. The next two days, mice were tested on the easy $35-\mathrm{cm}$ distance and then the challenging $20-\mathrm{cm}$ distance (Figure $4 \mathrm{~b}$ ).

The young/old pairing procedure applied to the young and old vehicle-treated groups was again successful in minimizing the effect of age on total exploration during the sample trials (young vehicle: $22.6 \pm 0.8 \mathrm{~s}$; old vehicle: $21.7 \pm 1.1 \mathrm{~s}$; old D-serine: $24.1 \pm 0.9 \mathrm{~s} ; \mathrm{F}(2,29)=$ 1.66, $p>0.10$; Group x Day interaction: $F(4,58)=1.14, p>0.10)$. 
D-serine had no effect on the high performances obtained in the 35-cm distance test (Group: $F(2,29)=0.14, p>0.10)$. On the challenging $20-\mathrm{cm}$ distance, there was a significant effect of group $(F(2,29)=6.80, p<0.004)$. D-serine-treated old mice performed nearly as well as young vehicle-treated mice and both groups performed significantly better than vehicletreated old mice. The severe deficit in the latter group was not related to sample exploration (Spearman rank test. $P=0.18, z=0.53, p=0.60$; all groups: $\rho=0.15, z=0.86, p=0.39$ ).

As a first approach to identify one possible mechanism involved in the beneficial effect of Dserine, we analyzed the impact of treatment on the survival of 4-week-old GCL cells in all mice (except for one young mouse, due to technical problems). As classically found, younger mice had higher mean $\mathrm{BrdU}^{+}$cell density than vehicle- or D-serine-treated older mice $(\mathrm{F}(2$, $28)=118.69, p<0.0001$, Figure $4 c)$. When only aged groups were considered, there was a small increase in $\mathrm{BrdU}^{+}$cells in the D-serine group that just reached significance $(t=2.14$, df $=19, \mathrm{p}=0.046)$. There was no correlation between $\mathrm{PS}$ performance and $\mathrm{BrdU}^{+}$cell density in young vehicle-treated mice, but we found an intriguing negative correlation for each aged group (Spearman rank test. 3-month vehicle: $\rho=-0.08, z=0.24, p=0.81 ; 18$-month vehicle: $\rho=-0.71, z=2.16, p=0.031 ; 18$-month D-serine: $\rho=-0.63, z=2.00, p=0.046)$.

\section{Discussion}

The main aim of the present study was to characterize the effect of aging on PS performance in C57BL/6J male mice. We first designed two PS tasks, as close as possible to those used in humans. These tasks were based on behavioral detection of subtle changes in object features and in spatial location of an object. We showed that i) PS performance was severely impaired in object and in spatial tasks at the age of 18 months; ii) spatial PS performance gradually declined between the ages of 3 and 21 months, with a significant deficit already detected at the age of 11 months; and iii) D-serine treatment fully rescued the performance of 18-month-old mice in the spatial PS task.

Before further discussing our results, it is necessary to consider whether the PS performance deficits of the older mice were due to impaired cognition or to non-cognitive age-related 
alterations. Firstly, it should be noted that PS performance deficit was still found in older mice after controlling for age-related decrease in sample exploration, either globally or individually across ages. Age-dependent decrease in motivation for novelty-driven object exploration is unlikely to have contributed, as young and aged groups were equally efficient in low-similarity trials on both object and spatial tasks. In addition, aged mice performed very similarly to young mice in the two spatial navigation tasks, especially in the water-maze task, which was shown by Yassine et al. (2013) to be critically dependent on vision. The same study showed that 21-month old blind mice from a mixed SJL (blind) and C57BL/6 genetic background performed efficiently in an object location task and in an object recognition task using objects of different shapes. Thus, subtle changes in the visual capacities of our aged mice may have marginally contributed to their deficits in the object PS task, but are very unlikely to be responsible for deficits in the spatial PS task. Lastly, the clear improvement in spatial PS performance obtained with D-serine treatment, without affecting other behavioral measures such as sample exploration, also pleads in favor of a cognitive deficit. Thus, age-related impairment in PS performance appears to be mainly due to altered cognitive processing rather than to defects in gross sensorimotor, visual or motivational aspects of the task.

To the best of our knowledge, this is the first study in a single animal model to show the deleterious effect of aging on PS ability for both object location and object features, as reported in humans (Stark et al., 2013; Reagh et al., 2014). Deficits in spatial PS performance in aged rodents mainly affect tasks based on place learning in a maze and object location discrimination learning in a touchscreen paradigm (Creer et al. 2010; Gracian et al., 2013). Deficits in object PS performance were previously reported in aged monkeys performing an object discrimination learning task and in aged rats performing a learning task or a two-trial task similar to ours (Burke et al., 2011; Johnson et al., 2017).

The present study also uniquely characterized the progression of spatial PS performance in mice throughout adulthood (from 3 to 21 months). Deficits were already significant at the age of 11 months, and the 21-month-old group performed at chance level. Interestingly, learning and memory performance in the Barnes maze and Morris water-maze tasks were spared in 
aged mice that were nevertheless severely impaired in the spatial PS task. The spatial PS task is quite different from spatial navigation tasks, which rely on extensive training and strong motivation to navigate toward a goal to escape from water or bright light; moreover, multiple training trials may allow older mice to learn the goal location as accurately as younger mice by the end of spatial navigation training. We concluded that these spatial navigation tasks were not sensitive enough to be affected by subtle age-related alterations such as those detected in the spatial PS task. Conversely, spatial PS abilities may not be essential for successful navigation based on easily identifiable distal cues (McHugh et al., 2007; Imielski et al., 2012). Taken together, the present results highlight the exceptional sensitivity of our spatial PS task to aging in C57BL/6J mice. It is noteworthy that the gradual decline in performance during mouse adulthood was reminiscent of that reported in humans from the ages of 20 to 89 years (see Figure $2 b$ in Stark et al., 2013). This striking similarity suggests a high degree of translational validity of our mouse model, and confirms the hypothesis of common age-dependent alterations in the mechanisms underlying PS across species (Burke et al., 2011).

Human fMRI studies have repeatedly shown an involvement of the DG-CA3 region in PS tasks taxing domains as different as object features and object location, as well as temporal or environmental information (Azab et al., 2014; Bakker et al., 2008; Kyle et al., 2015; Lacy et al., 2010; Reagh et al., 2014). More recently, it was suggested that interference resolution capacity increases while domain selectivity decreases as information proceeds along the ventral "object" stream (perirhinal cortex, lateral entorhinal cortex) and the dorsal "spatial" stream (parahippocampal cortex, medial entorhinal cortex), which both provide major input to the DG (Reagh and Yassa, 2014). In other words, upstream medial temporal lobe cortices may contribute to specific aspects of discrimination processes, while the downstream DGCA3 region may separate more multidimensional representations. This hypothesis could shed some light on a long-lasting debate in animal studies concerning the structure or structures involved in object PS ability. Several studies in rats showed that an intact perirhinal cortex was required to discriminate objects with overlapping features (Bussey et 
al., 2005; Bartko et al., 2007; but see Hales et al., 2015). Alternatively, interruption of objectrelated information flow along the ventral stream by perirhinal lesions may affect further steps in object discrimination processing. It is noteworthy that, to the best of our knowledge, only one animal study explored (and supported) the role of adult DG neurogenesis in an object PS task (Bolz et al., 2015). Thus, object PS deficits in aged animals have so far been mainly attributed to the deterioration of perirhinal functionality (Burke et al., 2011; Burke et al., 2012; Burke et al., 2014).

On the other hand, animal studies focusing on spatial and contextual aspects of PS ability are generally in favor of a key role of the dorsal DG and an involvement of local adult neurogenesis (e.g., Deng et al., 2013: Gilbert et al., 2001; Goodrich-Hunsaker et al., 2008; Sahay et al., 2011). The production of new DG neurons is known to decrease markedly with age (e.g., Ben Abdallah et al., 2010; Morgenstern et al., 2008). Our 18-month-old mice showed a much lower proportion of BrdU+ cells in the DG than 3-month-old counterparts. As stereological methods were not used here, it is possible that age-related volumetric changes in the DG could have contributed to this large age-related difference in BrdU+ cell density. Voluntary exercise was repeatedly reported to boost both neurogenesis and PS performance in young adult mice (Bolz et al., 2015; Creer et al., 2010). As further discussed below, the relationship between neurogenesis and PS performance may be affected by aging or put into question, as exercise does not necessarily improve both performance and neurogenesis in aged mice (Creer et al., 2010; Wu et al., 2015). The scarcity of studies investigating this issue in aged animal models emphasizes our limited knowledge of the neuronal changes underlying age-related deficits in PS performance.

D-serine is considered to be a promising therapeutic approach, with minimal side effects, for age-related cognitive decline (Mothet et al., 2015). In the present study, 1 week's pharmacological treatment with D-serine completely reversed the deficits of 18 -month-old mice in the spatial PS task. This finding is in agreement with an important role of dentate NMDAR in PS performance and in the maturation of young adult-born neurons (Kannangara et al, 2014; Kheirbek et al., 2012; McHugh et al., 2007; Sultan et al., 2013). As shown by 
Tashiro et al. (2006), activation of dentate NMDAR was also necessary for young neuron survival around 18 days after birth, a critical period covered by the D-serine treatment initiated 18 days after BrdU injection. Nevertheless, aged mice treated with D-serine showed a modest increase in the number of 4 -week-old BrdU+ cells in their dorsal GCL, which remained much lower than found in young mice. On the other hand, the D-serine-treated mice performed nearly as well as young mice in the spatial PS task. These findings do not support a simple quantitative relationship between number of 4-week-old GCL cells and PS performance. Moreover, the negative correlation between $\mathrm{BrdU}^{+}$cell density and spatial PS performance, specifically found in aged mice, appeared rather counterintuitive in this regard. This intriguing result should be confirmed by more detailed investigation of neurogenesis (e.g., phenotyping $\mathrm{BrdU}^{+}$cells and stereology). On the other hand, similar small increases in neurogenesis induced by other neurogenic treatments have been shown to improve other aspects of cognition in aged rodents (Gibbons et al., 2014; Pardo et al., 2016). Thus, a small increase in the number of dentate cells may trigger large-scale experience-dependent changes downstream the hippocampal network, due to the large impact of sparse dentate cell firing on the activity of CA3 pyramidal cells (Rebola et al, 2017). Alternatively, D-serine may have improved spatial PS performance by acting on the excitability and/or morphology of adult-born DG cells. Taken together, the present results suggest that the link between neurogenesis and PS performance, if any, is far more complicated than the current "the more the better" hypothesis (see also Wu et al., 2015). Therefore, alternative targets must be also be considered for the behavioral effect of $D$-serine, especially since chronic systemic administration is expected to induce a long-lasting increase in whole-brain D-serine contents (Bado et al., 2011). One possibility is that its beneficial effects may be mediated through NMDARs of other DG cells, or an upstream medial temporal lobe structure such as the entorhinal cortex. As a more specific approach, stimulation of local production and/or release of endogenous D-serine within the suspected structure of aged mice, with subsequent testing on the spatial PS task, could help resolve this anatomic issue. 
In conclusion, the present study showed that aged mice were severely impaired in both object and spatial PS tasks and that spatial PS deficits, which appear gradually as a function of age, can be reversed by a subchronic D-serine treatment. Our model of age-related deficits in two different PS tasks has the advantage of imposing minimal constraints on (aged) animal welfare. The model should be very helpful in identifying the specific changes in the medial temporal lobe which impair PS performance. Improving our knowledge on such issues is of even broader interest, since impairment in PS ability has been suggested to contribute to episodic memory deficit in normal and pathological aging, and in other human brain disorders (Das et al., 2014; Déry et al., 2015; Bakker et al, 2012; Stark et al., 2013).

\section{Funding and Disclosure:}

This work was supported by the RMT-TMO Offensive Science initiative, INTERREG IV Upper Rhine program (EU-funded project (ERDF) "Neurogenesis and Neuroprotection"), the France Alzheimer Association, Neurex, the University of Strasbourg, and the Centre National de la Recherche Scientifique (CNRS). All these funding sources had no involvement in any aspect of the study design, in the collection, analysis and interpretation of data, in the writing of the report or in the decision to submit the article for publication. The authors declare that they have no competing financial interests in relation to the work described.

\section{Acknowledgments:}

The authors thank Anne-Sophie Widling for preliminary tests useful to this project, and Olivier Bildstein and Georges Egesi for taking care of our young and aged mice. 


\section{REFERENCES}

Avellar M, Scoriels L, Madeira C, Vargas-Lopes C, Marques P, Dantas C, Manhães AC, Leite $H$, Panizzutti R. The effect of D-serine administration on cognition and mood in older adults. Oncotarget 2016;7:11881-8.

Azab M, Stark SM, Stark CE. Contributions of human hippocampal subfields to spatial and temporal pattern separation. Hippocampus 2014;24:293-302.

Bado P, Madeira C, Vargas-Lopes C, Moulin TC, Wasilewska-Sampaio AP, Maretti L, de Oliveira RV, Amaral OB, Panizzutti R. Effects of low-dose D-serine on recognition and working memory in mice. Psychopharmacology (Berl) 2011;218:461-70.

Bakker A, Kirwan CB, Miller M, Stark CE. Pattern separation in the human hippocampal CA3 and dentate gyrus. Science 2008;319:1640-2.

Bakker A, Krauss GL, Albert MS, Speck CL, Jones LR, Stark CE, Yassa MA, Bassett SS, Shelton AL, Gallagher M. Reduction of hippocampal hyperactivity improves cognition in amnestic mild cognitive impairment. Neuron. 2012 May 10;74(3):467-74.

Bartko SJ, Winters BD, Cowell RA, Saksida LM, Bussey TJ. Perirhinal cortex resolves feature ambiguity in configural object recognition and perceptual oddity tasks. Learn Mem 2007;14:821-32.

Becker S. Neurogenesis and pattern separation: time for a divorce. Wiley Interdiscip Rev Cogn Sci 2016. doi: 10.1002/wcs.1427.

Ben Abdallah NM, Slomianka L, Vyssotski AL, Lipp HP. Early age-related changes in adult hippocampal neurogenesis in C57 mice. Neurobiol Aging 2010;31:151-61.

Bolz L, Heigele S, Bischofberger J. Running improves pattern separation during novel object recognition. Brain Plasticity 2015;1:129-41.

Burke SN, Wallace JL, Hartzell AL, Nematollahi S, Plange K, Barnes CA. Age-associated deficits in pattern separation functions of the perirhinal cortex: a cross-species consensus. Behav Neurosci 2011;125:836-47. 
Burke SN, Hartzell AL, Lister JP, Hoang LT, Barnes CA. Layer V perirhinal cortical ensemble activity during object exploration: a comparison between young and aged rats. Hippocampus 2012;22:2080-93.

Burke SN, Maurer AP, Nematollahi S, Uprety A, Wallace JL, Barnes CA. Advanced age dissociates dual functions of the perirhinal cortex. J Neurosci 2014;34:467-80.

Bussey TJ, Saksida LM, Murray EA. The perceptual-mnemonic/feature conjunction model of perirhinal cortex function. Q J Exp Psychol B 2005;58:269-82.

Clelland CD, Choi M, Romberg C, Clemenson GD Jr, Fragniere A, Tyers P, Jessberger S, Saksida LM, Barker RA, Gage FH, Bussey TJ. A functional role for adult hippocampal neurogenesis in spatial pattern separation. Science 2009;325:210-3.

Conway MA. Episodic memories. Neuropsychologia 2009;47:2305-2313.

Creer DJ, Romberg C, Saksida LM, van Praag H, Bussey TJ. Running enhances spatial pattern separation in mice. Proc Natl Acad Sci USA 2010;107:2367-2372.

Das T, Ivleva El, Wagner AD, Stark CE, Tamminga CA. Loss of pattern separation performance in schizophrenia suggests dentate gyrus dysfunction. Schizophr Res 2014;159:193-7.

Deng W, Mayford M, Gage FH. Selection of distinct populations of dentate granule cells in response to inputs as a mechanism for pattern separation in mice. Elife 2013;20;2:e00312.

Déry N, Goldstein A, Becker S. A role for adult hippocampal neurogenesis at multiple time scales: A study of recent and remote memory in humans. Behav Neurosci 2015;129:43549.

Ennaceur A, Delacour J. A new one-trial test for neurobiological studies of memory in rats. 1: Behavioral data. Behav Brain Res 1988;31:47-59.

Ennaceur A, Neave N, Aggleton JP. Spontaneous object recognition and object location memory in rats: the effects of lesions in the cingulate cortices, the medial prefrontal cortex, the cingulum bundle and the fornix. Exp Brain Res 1997;113:509-19. 
Filali M, Lalonde R. The effects of subchronic D-serine on left-right discrimination learning, social interaction, and exploratory activity in APPswe/PS1 mice. Eur J Pharmacol 2013;701:152-8.

Gámiz F, Gallo M. Spontaneous object recognition memory in aged rats: Complexity versus similarity. Learn Mem 2012;19:444-8.

Gibbons TE, Pence BD, Petr G, Ossyra JM, Mach HC, Bhattacharya TK, Perez S, Martin SA, McCusker RH, Kelley KW, Rhodes JS, Johnson RW, Woods JA. Voluntary wheel running, but not a diet containing (-)-epigallocatechin-3-gallate and $\beta$-alanine, improves learning, memory and hippocampal neurogenesis in aged mice. Behav Brain Res 2014;272:131-40.

Gilbert PE, Kesner RP, Lee I. Dissociating hippocampal subregions: double dissociation between dentate gyrus and CA1. Hippocampus 2001;11:626-36.

Goodrich-Hunsaker NJ, Hunsaker MR, Kesner RP. The interactions and dissociations of the dorsal hippocampus subregions: how the dentate gyrus, CA3, and CA1 process spatial information. Behav Neurosci 2008;122:16-26.

Gracian EI, Shelley LE, Morris AM, Gilbert PE. Age-related changes in place learning for adjacent and separate locations. Neurobiol Aging 2013;34:2304-9.

Greenberg GD, Howerton CL, Trainor BC. Fighting in the home cage: Agonistic encounters and effects on neurobiological markers within the social decision-making network of house mice (Mus musculus). Neurosci Lett 2014;566:151-5.

Groves JO1, Leslie I, Huang GJ, McHugh SB, Taylor A, Mott R, Munafò M, Bannerman DM, Flint J. Ablating adult neurogenesis in the rat has no effect on spatial processing: evidence from a novel pharmacogenetic model. PLoS Genet 2013;9:e1003718.

Hales JB, Broadbent NJ, Velu PD, Squire LR, Clark RE. Hippocampus, perirhinal cortex, and complex visual discriminations in rats and humans. Learn Mem 2015;22:83-91.

Imielski Y, Schwamborn JC, Lüningschrör P, Heimann P, Holzberg M, Werner H, Leske O, Püschel AW, Memet S, Heumann R, Israel A, Kaltschmidt C, Kaltschmidt B. Regrowing the adult brain: NF-KB controls functional circuit formation and tissue homeostasis in the dentate gyrus. PLoS One 2012;7:e30838. 
Johnson SA, Turner SM, Santacroce LA, Carty KN, Shafiq L, Bizon JL, Maurer AP, Burke SN. Rodent age-related impairments in discriminating perceptually similar objects parallel those observed in humans. Hippocampus 2017;27:759-776.

Kannangara TS, Bostrom CA, Ratzlaff A, Thompson L, Cater RM, Gil-Mohapel J, Christie BR. Deletion of the NMDA receptor GluN2A subunit significantly decreases dendritic growth in maturing dentate granule neurons. PLoS One 2014;9:e103155.

Kheirbek MA, Tannenholz L, Hen R. NR2B-dependent plasticity of adult-born granule cells is necessary for context discrimination. J Neurosci 2012;32:8696-702.

Kyle CT, Stokes JD, Lieberman JS, Hassan AS, Ekstrom AD. Successful retrieval of competing spatial environments in humans involves hippocampal pattern separation mechanisms. Elife 2015;27;4.

Lacy JW, Yassa MA, Stark SM, Muftuler LT, Stark CE. Distinct pattern separation related transfer functions in human $\mathrm{CA} 3 /$ dentate and CA1 revealed using high-resolution $\mathrm{fMRI}$ and variable mnemonic similarity. Learn Mem 2010;18:15-8.

Leutgeb JK, Leutgeb S, Moser MB, Moser El. Pattern separation in the dentate gyrus and CA3 of the hippocampus. Science 2007;315:961-6.

Levin R, Dor-Abarbanel AE, Edelman S, Durrant AR, Hashimoto K, Javitt DC, Heresco-Levy U. Behavioral and cognitive effects of the N-methyl-D-aspartate receptor co-agonist Dserine in healthy humans: initial findings. J Psychiatr Res 2015;61:188-95.

McHugh TJ, Jones MW, Quinn JJ, Balthasar N, Coppari R, Elmquist JK, Lowell BB, Fanselow MS, Wilson MA, Tonegawa S. Dentate gyrus NMDA receptors mediate rapid pattern separation in the hippocampal network. Science 2007;317:94-9.

Marr D. Simple memory: a theory for archicortex. Philos Trans R Soc Lond B Biol Sci $1971 ; 262: 23-81$.

Marrone DF, Adams AA, Satvat E. Increased pattern separation in the aged fascia dentata. Neurobiol Aging 2011;32:2317.e23-2332.e23. 
Moreau PH, Cosquer B, Jeltsch H, Cassel JC, Mathis C. Neuroanatomical and behavioral effects of a novel version of the cholinergic immunotoxin mu p75-saporin in mice. Hippocampus 2008;18:610-22.

Morgenstern NA1, Lombardi G, Schinder AF. Newborn granule cells in the ageing dentate gyrus.J Physiol 2008;586:3751-7.

Mothet JP, Le Bail M, Billard JM. Time and space profiling of NMDA receptor co-agonist functions. J Neurochem 2015;135:210-25.

Nakashiba T, Cushman JD, Pelkey KA, Renaudineau S, Buhl DL, McHugh TJ, Rodriguez Barrera V, Chittajallu R, Iwamoto KS, McBain CJ, Fanselow MS, Tonegawa S. Young dentate granule cells mediate pattern separation, whereas old granule cells facilitate pattern completion. Cell 2012;149:188-201.

Pardo J, Uriarte M, Cónsole GM, Reggiani PC, Outeiro TF, Morel GR, Goya RG. Insulin-like growth factor-I gene therapy increases hippocampal neurogenesis, astrocyte branching and improves spatial memory in female aging rats. Eur J Neurosci 2016;44:2120-8.

Reagh ZM, Roberts JM, Ly M, DiProspero N, Murray E, Yassa MA. Spatial discrimination deficits as a function of mnemonic interference in aged adults with and without memory impairment. Hippocampus 2014;24:303-14.

Reagh ZM, Yassa MA. Object and spatial mnemonic interference differentially engage lateral and medial entorhinal cortex in humans. Proc Natl Acad Sci USA 2014;111:E4264-73.

Rebola N, Carta M, Mulle C. Operation and plasticity of hippocampal CA3 circuits: implications for memory encoding. Nat Rev Neurosci 2017;18:208-220.

Rolls ET, Kesner RP. A computational theory of hippocampal function, and empirical tests of the theory. Prog Neurobiol 2006;79:1-48.

Sahay A, Scobie KN, Hill AS, O'Carroll CM, Kheirbek MA, Burghardt NS, Fenton AA, Dranovsky A, Hen R. Increasing adult hippocampal neurogenesis is sufficient to improve pattern separation. Nature 2011;472:466-70.

Schneider CA, Rasband WS, Eliceiri KW. NIH Image to ImageJ: 25 years of image analysis. Nat Methods 2012;9:671-5. 
Snyder JS, Choe JS, Clifford MA, Jeurling SI, Hurley P, Brown A, Kamhi JF, Cameron HA. Adult-born hippocampal neurons are more numerous, faster maturing, and more involved in behavior in rats than in mice. J Neurosci 2009;29:14484-95.

Stark SM, Yassa MA, Stark CE. Individual differences in spatial pattern separation performance associated with healthy aging in humans. Learn Mem 2010;17:284-8.

Stark SM, Yassa MA, Lacy JW, Stark CE. A task to assess behavioral pattern separation (BPS) in humans: Data from healthy aging and mild cognitive impairment. Neuropsychologia 2013;51:2442-9.

Sultan S, Gebara EG, Moullec K, Toni N. D-serine increases adult hippocampal neurogenesis. Front Neurosci 2013;7:155.

Tashiro A, Sandler VM, Toni N, Zhao C, Gage FH. NMDA-receptor-mediated, cell-specific integration of new neurons in adult dentate gyrus. Nature 2006;442:929-33.

Toner CK, Pirogovsky E, Kirwan CB, Gilbert PE. Visual object pattern separation deficits in nondemented older adults. Learn Mem 2009;16:338-42.

Tronel S, Belnoue L, Grosjean N, Revest JM, Piazza PV, Koehl M, Abrous DN. Adult-born neurons are necessary for extended contextual discrimination. Hippocampus 2012;22:292-98.

Veyrac A, Gros A, Bruel-Jungerman E, Rochefort C, Kleine Borgmann FB, Jessberger S, Laroche S. Zif268/egr1 gene controls the selection, maturation and functional integration of adult hippocampal newborn neurons by learning. Proc Natl Acad Sci U S A 2013;110:7062-7.

Wu MV, Luna VM, Hen R. Running rescues a fear-based contextual discrimination deficit in aged mice. Front Syst Neurosci 2015;9:114.

Yassa MA, Mattfeld AT, Stark SM, Stark CE. Age-related memory deficits linked to circuitspecific disruptions in the hippocampus. Proc Natl Acad Sci USA 2011;108:8873-78.

Yassine N, Lazaris A, Dorner-Ciossek C, Després O, Meyer L, Maitre M, Mensah-Nyagan AG, Cassel JC, Mathis C. Detecting spatial memory deficits beyond blindness in tg2576 Alzheimer mice. Neurobiol Aging 2013;34:716-30. 
Figure Legends

Figure 1: Behavioral novelty responses to a wide range of object displacements in 6-monthold C57BL/6J mice. a) Each group, A and B, was tested on three distance categories: short, medium and long displacement of the object between sample trial and test trial. b) Test trial performances for each group and for each distance category are shown as mean ( \pm SEM) percentage time spent exploring the displaced object during the first $3 \mathrm{~min}$. NK post-hoc test: ${ }^{*} p<0.05$ and ${ }^{* *} p<0.01$ versus medium-distance performance within a group. Student $t-$ test: $\$ p<0.05$ and $\$ \$ p<0.001$ versus $50 \%$ chance level.

a

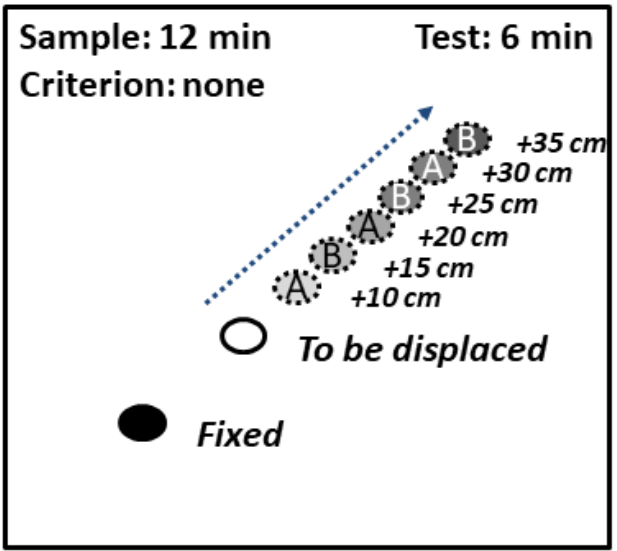

b

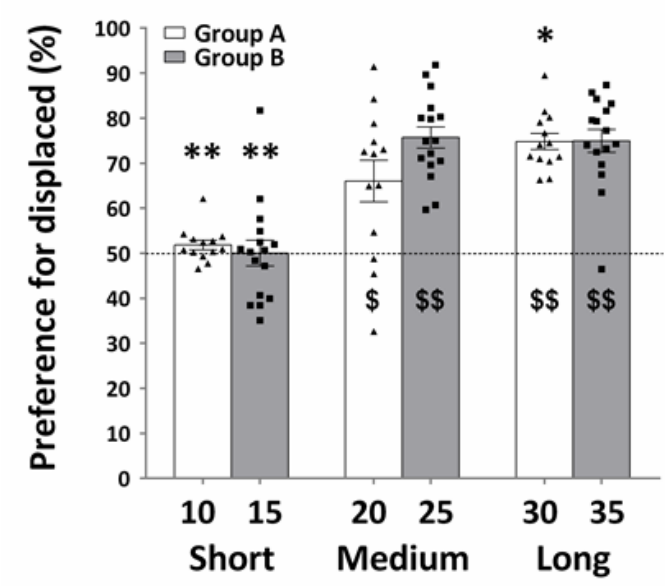


Figure 2: Age effect on behavioral novelty responses to object displacements in C57BL/6J mice. a) Performances of 6- and 20-month-old mice on $10 \mathrm{~cm}, 20 \mathrm{~cm}$ and $35 \mathrm{~cm}$ displacement distances. Aged mice were impaired on the challenging 20-cm distance compared to young mice (NK post-hoc test: ${ }^{*} p<0.05$ ). b) Performances of independent groups of mice tested on the 20 -cm distance at the ages of $3,7,11,16$ and 21 months (NK post-hoc test: ${ }^{*} p<0.05$ and ${ }^{*} p<0.01$ versus the 3 -month-old group; $\S p<0.05$ versus the 7-month-old group). Data are shown as mean ( \pm SEM) percentage time spent exploring the displaced object during the first 3 min of the test trial. Student $t$ test for both graphs: $\$ p<$ 0.05 and $\$ \$ p<0.001$ versus $50 \%$ chance level.

Sample: $15 \mathrm{~min}$ Criterion: none

Test: 6 min

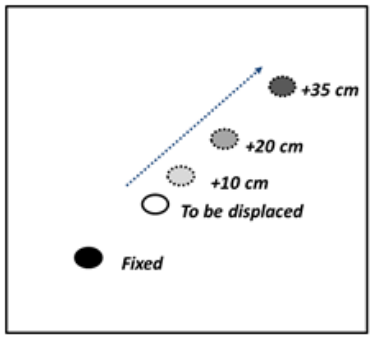

b
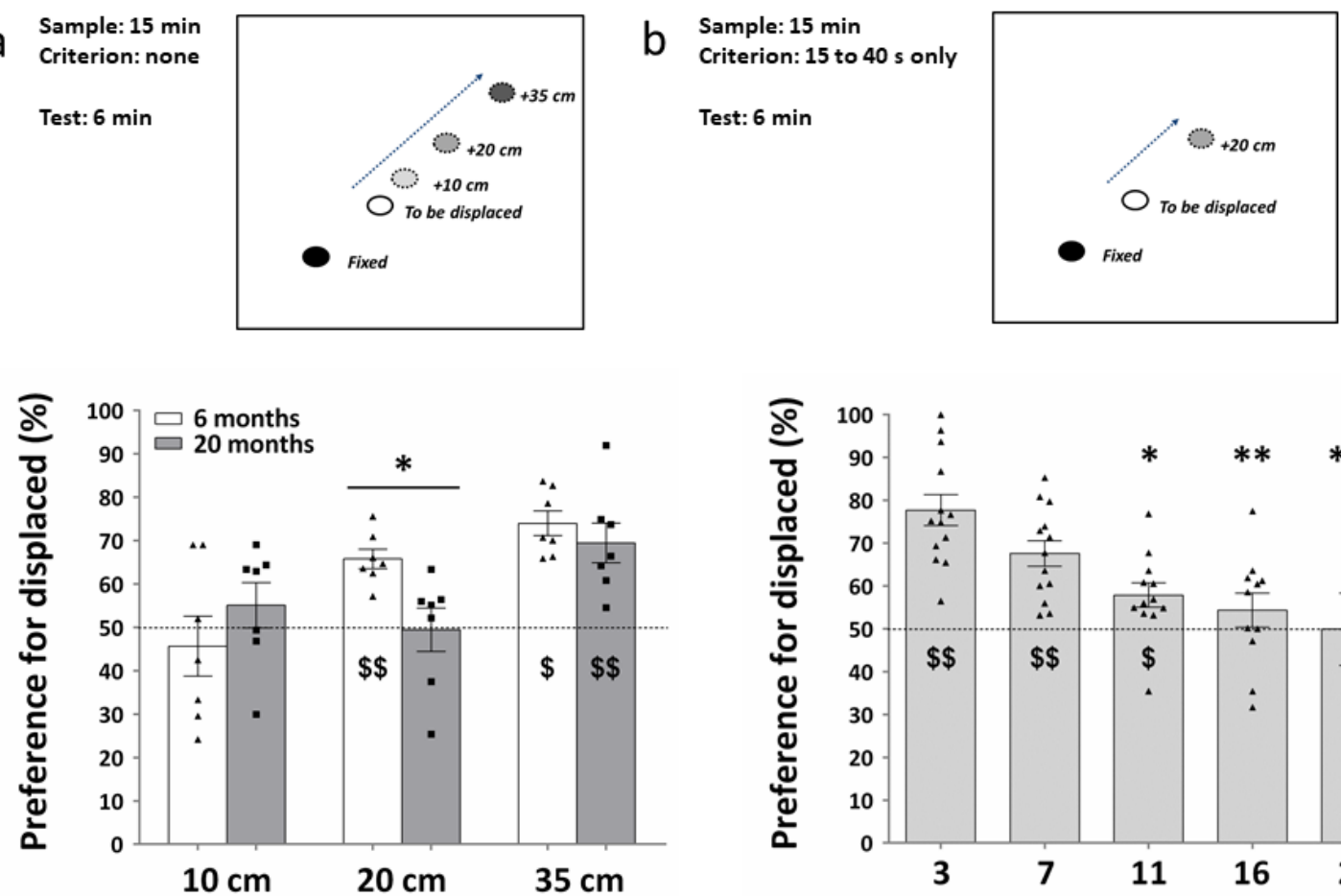

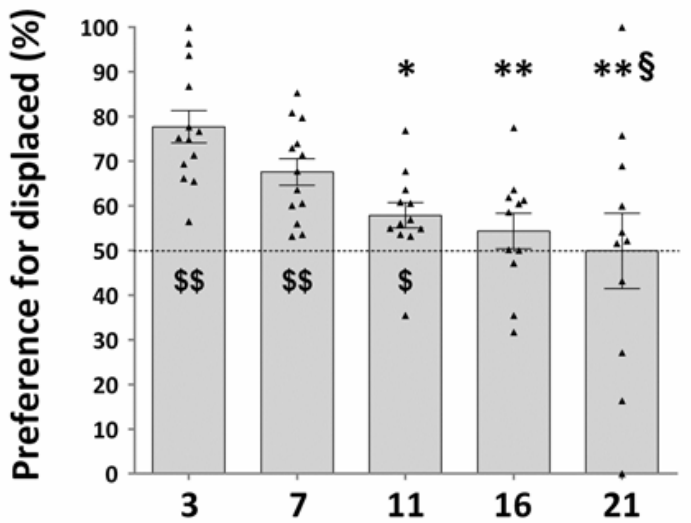

Age (months) 
Figure 3: Age effect on object pattern separation performance in C57BL/6J mice. a) Pictures of the two sets of familiar objects with corresponding high- and low-similarity objects. b) Performance of 4- and 18-month-old mice for new objects with high and low similarities with the familiar object. Data are shown as mean ( \pm SEM) percentage time spent exploring the new object during the 10 -min test trial. NK post-hoc test: ${ }^{* *} p<0.0005$ aged group versus young group; $\S \S p<0.0005$ versus performance on low-similarity trial. Student $t$ test: $\$ \$ p<$ 0.0001 versus $50 \%$ chance level.

a
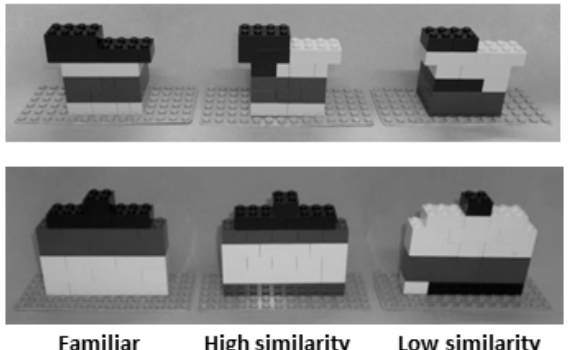

b Sample: $10 \mathrm{~min}$ Criterion: young-old pairing

Test: $10 \mathrm{~min}$
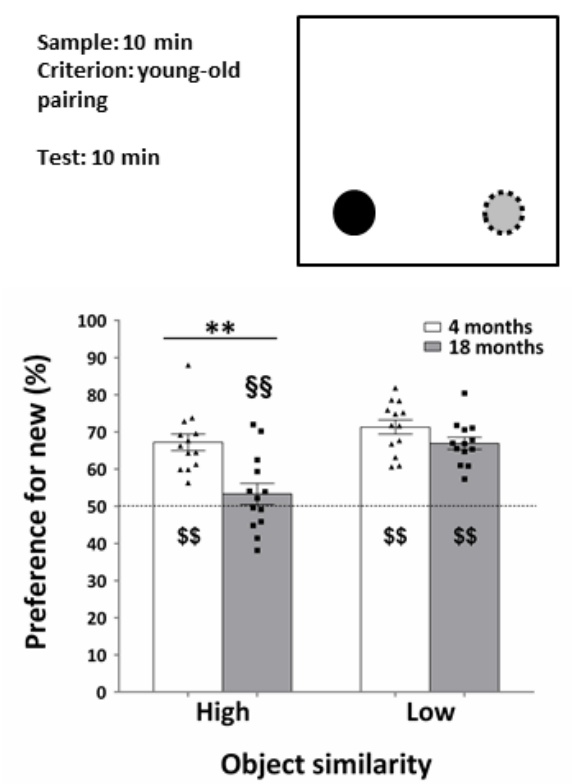
Figure 4: Effects of D-serine on novelty detection performance in 18-month-old C57BL/6J mice. a) Daily D-serine or vehicle treatment was applied 18 to 24 days after BrdU injection and before the 4-day behavioral test period. b) Performances were first determined on the easy $35-\mathrm{cm}$ distance and then on the challenging $20-\mathrm{cm}$ distance in 3-month-old mice treated with vehicle and in 18-month-old mice treated with vehicle or D-serine $(50 \mathrm{mg} / \mathrm{kg}, \mathrm{IP})$. Data are shown as mean $( \pm$ SEM) percentage time spent exploring the displaced object during the first 3 min of the test trial. NK post-hoc test: * $p<0.05$ versus 18 -month D-serine group, ${ }^{* *} p<0.01$ versus 3 -month vehicle group. Student t test: $\$ \$ p<0.001$ versus 50\% chance level. c) Representative micrographs of one section immunostained for BrdU for each group (x10 for micrographs, $x 40$ for inserts). The graph shows the effect of D-serine on the mean ( \pm SEM) 4-week-old $\mathrm{BrdU}^{+}$cell count per $10^{-2} \mathrm{~mm}^{3} \mathrm{GCL}$ volume for each group. This particular scale was chosen as mean GCL volume per section was $1.09 \pm 0.0310^{-2} \mathrm{~mm}^{3}$. * $\mathrm{p}$ $<0.05$ versus 18 -month vehicle group. ${ }^{* *} p<0.01$ versus each 18 -month group.

a

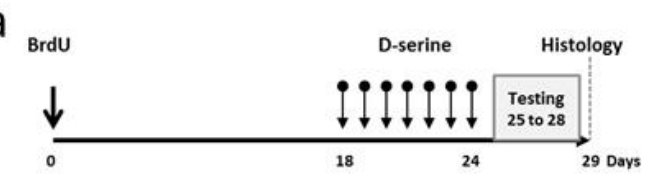

b
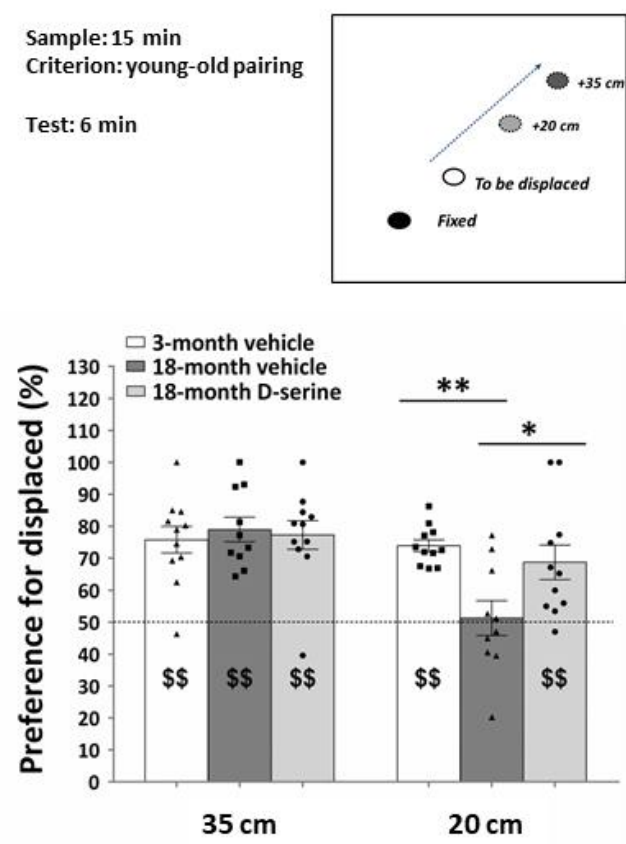

C
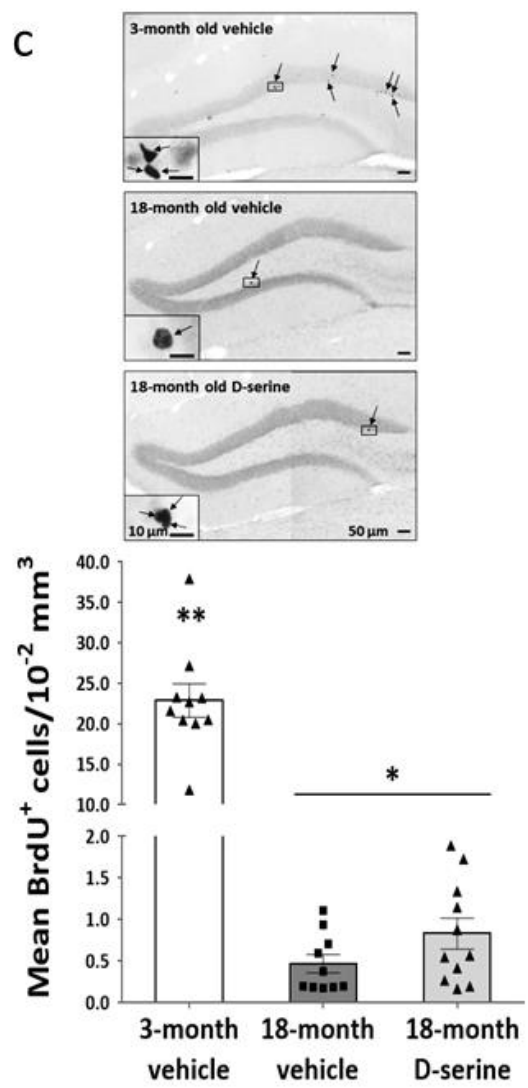


\section{Supplemental methods}

Standard spatial navigation tasks. The Morris water maze task was performed as described in Moreau et al (2008) except that the water maze consisted of a 150-cm diameter pool (Ugo Basile, Italy). Mice were trained for 5 days (4 trials/day) to find a submerged platform in the North-East quadrant. After a 7-day delay they received a 60-s probe trial with no platform. The Barnes maze task was performed as described in Moreau et al (2008) except that the maze was a 120-cm diameter board with 20 holes (Ugo Basile, Italy). Mice were trained for 5 days ( 3 trials/day) to find the fixed target hole which lead to their home cage and after a 28-day delay they received a 120-s probe trial with no hole connected to the home cage. After each trial, the board was wiped and rotated to avoid odor-based strategies. For each trial, the order of hole-visits was recorded to calculate the number of errors (visits to non-target holes) and the percentage of visit to four equidistant holes including the target hole during the probe trial. Trajectories in both tasks were analyzed for latency and distance traveled to reach targets using a video-tracking system (ANY-maze 4.3; Ugo Basile, Italy).

\section{Supplemental data}

Legend of Figure 1S: Performances of 6-month and 20-month old C57BL/6J mice in standard spatial navigation tasks. Barnes maze task: a) training latencies to reach the target hole was similar in aged and young mice (Latency: Day $F(4,48)=24.22, p<0.0001$; Error: Day $F(4,48)=3.81, p<0.009$; Distance: Day $F(4,48)=10.97, p<0.0001$; Data not shown for errors and distance; no effect of Age or Age x Day interaction). b) After a 28-day delay, young and old mice performed similarly in the probe test. Note that one old mice died before completing the probe. For both groups, the percentage of visits to the target hole was higher than the mean percentage of visits to the opposite hole and those located $90^{\circ}$ on the right and the left of the target hole (Hole: $F(3,33)=12.28, p<0.0001$; no Age effect or Age $x$ Hole interaction). Water maze task: c) training latencies to reach the platform were similar between young and aged mice (Latency: Day $F(4,48)=9.75, p<0.0001$; Distance (data not shown): Day $F(4,48)=5.05, p<0.002$; no effect of Age or Age $x$ Day interaction; Speed 
(data not shown): no effect of Age, Day or or Age x Day interaction). d) Both groups spent more time in the target quadrant compared to other quadrants (Quadrant: $F(3,36)=20.73, p$ $<0.0001$; no effect of Age or Age $x$ Quadrant interaction). Data are shown as mean + SEM. * $p<0.05$ versus the mean of other holes (b) or quadrants (d). $p<0.05$ and $p<0.001$ versus $5 \%$ chance level (b) and 15 s chance level (d). 

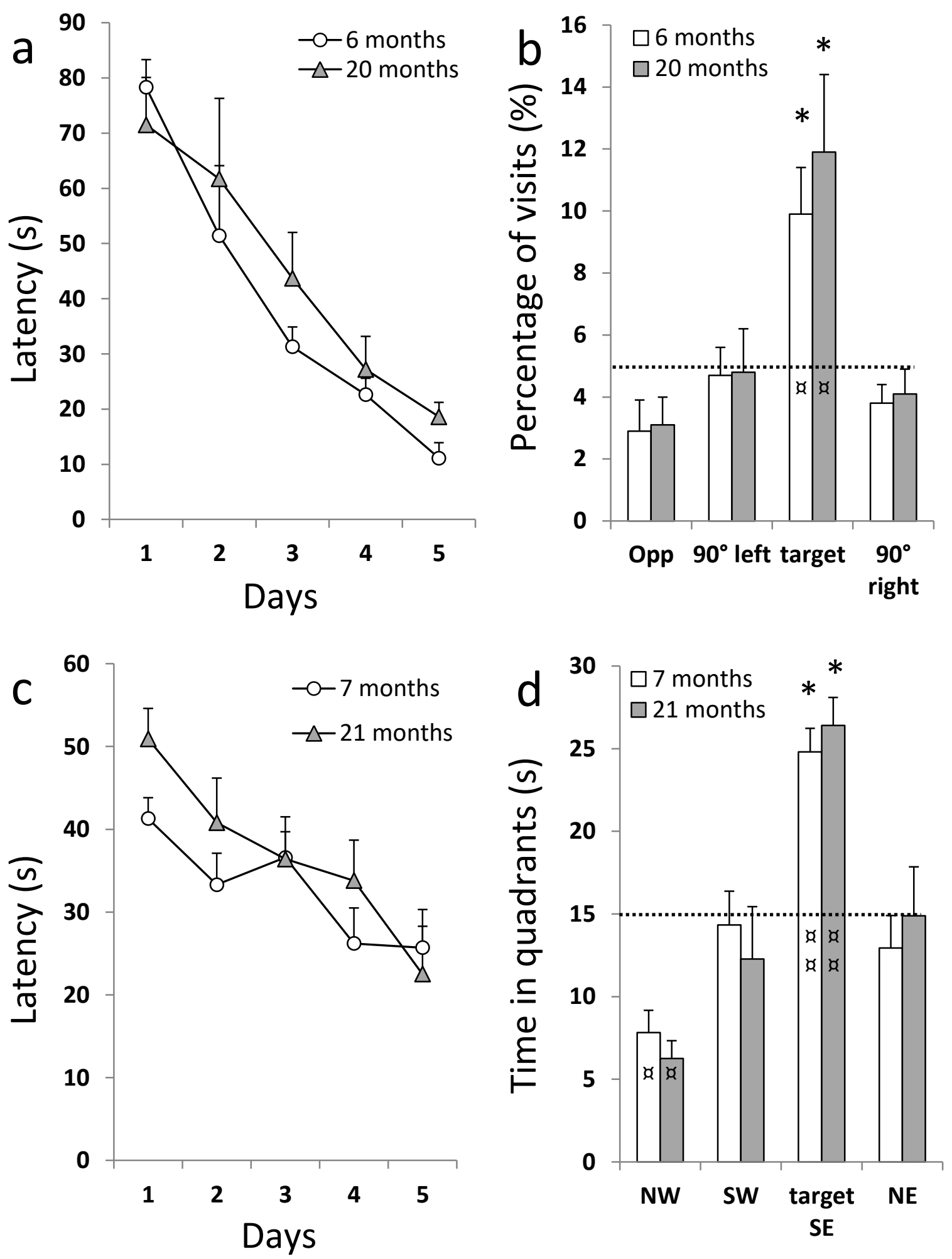